\title{
Transição, praxe e variáveis académicas e familiares. Estudos na Universidade
}

\author{
dos Açores
}

\section{Transition, hazing and academic and family variables. Studies at the University of the Azores}

\author{
Osvaldo Silva *, Suzana Nunes Caldeira *, Áurea Sousa**, Maria Mendes***, Maria José D. Martins**** \\ *Universidade dos Açores, Centro Interdisciplinar de Ciências Sociais - CICS.UAc/CICS.NOVA.UAc, **Universidade dos Açores, \\ CEEAplA, ***Universidade dos Açores, GaPEOS, ****Instituto Politécnico de Portalegre, UIDEF-IEUL, CIEP-UE.
}

\begin{abstract}
Resumo
Investigaram-se variáveis académicas e familiares, procurando-se descortinar a sua relação com o posicionamento dos estudantes face às vivências da praxe, a nível da sua integração académica. 163 estudantes do $1 .^{\circ}$ ano do Ensino Superior responderam a um questionário e à subescala "Integração" do Questionário de Vivências da Praxe (QVP). O "Tempo de estudo" foi a única variável, entre as estudadas, que apresentou uma associação estatisticamente significativa com o "Posicionamento face às vivências da praxe". O rendimento académico é influenciado por diversas variáveis, entre as quais o "Sexo", a "Posição da opção de escolha do curso" e o "Tempo de estudo".

Palavras clave: Ensino superior; vivência da praxe, integração académica.
\end{abstract}

\begin{abstract}
Some academic and family variables were investigated, in order to perceive their relationship with the positioning of the university students in relation to the role of the hazing practices, in terms of their academic integration. 163 students from Higher Education answered to a questionnaire and to the Hazing Living Experiences Questionnaire (QVP). "Study time" was the only variable, among those studied, that presented a statistically significant association with the "Positioning in relation to the hazing practices". Moreover, the academic performance of the students is influenced by several variables, among which are the "Gender", "Position of choice of course" and "Time of study".

Keywords: Higher education; practice hazing; academic integration.
\end{abstract}

\section{Introdução}

A transição para o Ensino Superior e a adaptação dos estudantes a esta nova etapa formativa tem sido um tema investigado já há vários anos nas instituições portuguesas. Como se encontra descrito em Soares, Pinheiro e Canavarro (2015), esta é uma das transições mais desafiantes vivenciadas pelo indivíduo durante o seu trajeto académico, tendo em conta que comporta mudanças de carácter educativo, as quais estão, em muitos casos, associadas a mudanças noutras dimensões da vida. Isto é, o confronto com uma nova instituição, com um ensino menos estruturado e com métodos de estudo e aprendizagem mais exigentes do ponto de vista da autonomia, pode ocorrer em simultâneo com a separação de família e amigos, a necessidade de estabelecimento de novas relações sociais, a assunção de responsabilidades no âmbito da gestão da vida diária, desde a gestão e organização do tempo, à gestão de recursos financeiros e de outros aspetos ligados à sobrevivência. A par de todos estes desafios, há ainda a atender às exigências psicológicas que ocorrem neste período associadas à passagem da adolescência para a idade adulta (Andrade, 2016).

Não obstante todas essas mudanças que marcam a entrada no Ensino Superior, a investigação informa que a maioria dos estudantes é bem-sucedida na sua adaptação a essa entrada (e.g., Soares, Pinheiro \& Canavarro, 2015). Um dos fatores a considerar para esse resultado parece ser o modo como aqueles se sentem acolhidos no novo meio, uma vez que "(...) a existência ou disponibilidade de pessoas em quem podemos confiar, que nos deixam entender que se preocupam connosco, que nos valorizam e que gostam de nós" (Sarason, Levine, Basham \& Sarason, 1983, p.127) exerce um efeito harmonizador num período em que os desafios emocionais, sociais, académicos e institucionais imperam. E neste contexto, as praxes, enquanto ritual de receção e acolhimento dos novos estudantes no Ensino Superior, parecem ser consideradas, por muitos deles, como um processo atrativo, que facilita a transição e que contribui para uma adaptação positiva (Costa, Dias, Dias, Souza \& Canela, 2013; Pimentel, Mata \& Pereira, 2012).

$\mathrm{O}$ caso dos estudantes da Universidade dos Açores parece encontrar-se nesta situação, uma vez que, em vários estudos realizados, a proporção dos inquiridos que afirmam estar de acordo com as atividades de praxe é superior ou equivalente à dos que declaram discordar das mesmas (e.g., Botelho, Mendes, Caldeira, Silva, Martins \& Carvalho, 2015; Caldeira, Silva, Mendes, Martins, Miranda \& Silva, 2017; Silva, Caldeira; Mendes \& Botelho, 2014). Embora o nível de adesão a este tipo de atividade não seja particularmente elevado 
(Silva, Caldeira, Mendes, Botelho \& Martins, 2015), os estudantes referem que as praxes são divertidas e engraçadas, proporcionando momentos de convívio, de socialização e de integração (Martins, Caldeira, Silva, Mendes \& Botelho, 2015). Assim, será expectável que a vivência das praxes de forma positiva tenha implicações favoráveis em distintas vertentes dessa integração.

Uma das vertentes situa-se no domínio académico. Este é um tópico particularmente relevante no caso dos Açores, onde, de acordo com estatísticas regionais, 64\% dos alunos ficam detentores apenas do Ensino Básico e $32 \%$ têm só o $1 .^{\circ}$ ciclo (Direção Regional da Educação, 2015). Acresce que a Universidade dos Açores, por se tratar de uma instituição de pequena dimensão, na sua oferta formativa não contempla todas as áreas possíveis de formação. Tal situação pode ocasionar que os estudantes que nela ingressam renunciem, voluntariamente ou por força de outras circunstâncias, às suas primeiras escolhas. Este aspeto poderá ter implicações a nível dos comportamentos de estudo e adesão às atividades curriculares, com repercussões no rendimento académico alcançado.

Outra vertente reporta-se ao enquadramento familiar de origem, nomeadamente ao facto de os estudantes provirem de contextos cujos pais, ou familiares próximos, frequentaram, ou não, o Ensino Superior. Isto é, os estudantes estarem na condição de estudantes de primeira geração ou de estudantes de geração contínua. Resultados obtidos nos Açores indicam que o sentimento de relação positiva com a praxe é prevalecente em estudantes oriundos de um enquadramento familiar mais favorecido, em termos da escolaridade dos seus progenitores (Caldeira, Silva, Mendes \& Botelho, 2016), sendo, no entanto, reconhecido que esta é uma região do país com défice educacional há já várias gerações (Silva \& Sousa, 2015). Esta situação fará com que muitos dos estudantes que fazem a sua formação nos Açores sejam, sobretudo, estudantes de primeira geração de universitários nas suas famílias, situação que, de acordo com a literatura científica, os coloca face a um desafio maior relativamente aos que são de geração contínua (Araújo, Santos, Noronha, Zanon, Ferreira, Casanova \& Almeida, 2016). Os estudantes de primeira geração, por um lado tendem a alcançar notas mais baixas e a abandonar mais os estudos, em virtude de constrangimentos económicos, sociais e culturais que enfrentam (Stephens, Fryberg, Markus, Johnson \& Covarrubias, 2012) mas, por outro lado, também ganham resiliência, em virtude de serem muito comprometidos e envolvidos com a sua formação, pois entendem que esta é a principal forma de mobilidade social ascendente (Schuh, 2017).

Assim, neste trabalho primeiramente exploram-se variáveis relativas ao domínio académico, traduzidas pelo "Rendimento académico", a "Posição da opção de escolha do curso" e o "Tempo de estudo", e uma variável de índole familiar, designadamente o "Enquadramento familiar de origem", procurando-se perceber a relação destas variáveis com o "Posicionamento face às vivências da praxe" a nível da integração académica. Em seguida, e considerando que o "Rendimento académico" pode também ser tomado como um indicador da qualidade de adaptação e sucesso dos estudantes, não obstante o amplo reconhecimento da natureza multifacetada destes constructos (Soares, Pinheiro \& Canavarro, 2015), aprofundam-se os estudos referentes àquela variável.

\section{Procedimentos metodológicos}

Participaram neste estudo 163 estudantes do $1 .^{\circ}$ ano de diversas áreas científicas da Universidade dos Açores. A recolha de dados foi efetuada através de um protocolo que continha uma parte relativa a dados pessoais, académicos e familiares, de onde se salientam, para além do "Sexo", as variáveis "Rendimento académico", "Posição da opção de escolha do curso" e "Tempo de estudo". Nesta parte cada estudante indicou, ainda, qual o seu "Enquadramento familiar de origem", em termos do tipo de estudos dos progenitores. $\mathrm{O}$ protocolo continha ainda o Questionário de Vivências da Praxe (QVP), estudado para a população portuguesa por Vieira em 2013, o qual é definido por 18 itens, cujas opções de resposta variam de 1 (discordo totalmente) a 5 (concordo totalmente).

A variável "Rendimento académico" foi obtida através do cálculo da média das notas, declaradas por cada um dos estudantes, nas unidades curriculares do $1^{\circ}$ semestre. A variável "Posição da opção de escolha do curso" visou aferir se o curso frequentado pelo estudante foi ou não o escolhido como $1^{\text {a }}$ opção. A variável "Tempo de estudo" (indicada em horas) foi recodificada em três categorias, as quais são apresentadas na Tabela 1, na secção Resultados. A variável "Enquadramento familiar de origem" foi codificada em duas categorias, primeira geração e geração contínua, conforme pelo menos um dos progenitores fosse detentor, ou não, de formação a nível do Ensino Superior. Quanto ao QVP, foram calculadas, para cada um dos estudantes, as pontuações totais obtidas na sua subescala "Integração", a partir das quais se apurou a variável "Posicionamento face às vivências da praxe" a nível da sua integração académica em duas categorias (Negativo e Positivo). O ponto de corte para a definição das categorias foi as pontuações serem, ou não, inferiores ao ponto médio do intervalo de variação desta subescala. A consistência interna dos itens da subescala "Integração" do QVP na amostra utilizada é, de acordo com o valor (0.944) obtido para o coeficiente alfa de Cronbach, muito boa.

Para se explorar o comportamento das variáveis académicas ("Rendimento académico", "Posição da opção de escolha do curso" e "Tempo de estudo") e familiares ("Enquadramento familiar de origem"), e perceber a sua relação com o "Posicionamento face às vivências da praxe", a nível da integração académica, foram utilizados diversos procedimentos estatísticos: teste de Kolmogorov-Smirnov, para se averiguar a normalidade das distribuições; teste $t$ se Student, para comparação de duas médias populacionais (caso de duas amostras independentes); Análise de Variância (ANOVA) a um fator, para testar a hipótese de igualdade de médias, no caso de três ou mais amostras 
independentes; teste de independência do qui-quadrado, utilizado com vista a identificarem-se eventuais associações entre algumas variáveis, do domínio académico e familiar, e o "Posicionamento face às vivências da praxe"; Análise de Variância Fatorial, designadamente a ANOVA a cinco fatores, efetuada essencialmente com o intuito de aprofundar o estudo da variável "Rendimento académico"; e método não hierárquico das $k$-médias ( $k$-means), considerando uma partição em três classes (clusters), de forma a maximizar as diferenças entre os estudantes, no que se refere ao rendimento académico (média das notas), utilizado com vista a conhecer os perfis dos estudantes incluídos em cada um dos clusters.

\section{Resultados}

Passa-se a descrever o comportamento das variáveis académicas e familiar para, em seguida, se procurar perceber a sua relação com o posicionamento face à praxe, em termos da integração académica.

Os resultados indicam que o "Rendimento académico" dos inquiridos é satisfatório (média $=13.12$, mediana=13, moda=12.5). Quanto à "Posição da opção de escolha do curso", a maioria dos respondentes indicou frequentar o curso $(81.3 \%$ ) [e a universidade $(91.3 \%)$ ] correspondente à sua $1^{\mathrm{a}}$ opção, estando maioritariamente $(80.7 \%)$ inscritos pela primeira vez no Ensino Superior. Em termos de "Tempo de estudo", o número de horas, por semana, oscila entre 0 (mínimo) e 34 (máximo), com um valor médio de 10.12. No referente à variável "Enquadramento familiar de origem", a maioria dos estudantes $(79.1 \%)$ encontra-se na situação de primeira geração, enquanto uma minoria (20.9\%) tem pelo menos um dos progenitores com frequência do Ensino Superior.

No respeitante ao "Posicionamento face às vivências da praxe", a maioria dos inquiridos $(71.9 \%)$ expressouse favoravelmente em relação ao papel daquelas a nível da sua integração académica (QVP-Integração). Relacionando esta variável com as anteriormente descritas, observa-se:

- que os resultados da aplicação do teste $t$ de Student, para o caso de duas amostras independentes, indicam não existirem diferenças estatisticamente significativas $(p>0.05)$ entre os estudantes que mostraram um posicionamento positivo face à praxe e os que revelaram um posicionamento negativo, no respeitante ao "Rendimento académico", embora os primeiros tenham indicado classificações menos expressivas (12.7) do que os segundos (13.6);

- com base no teste de independência do quiquadrado, não existir associação estatisticamente significativa entre estudantes que se posicionam diferentemente (positiva $v s$ negativa) face à praxe e a "Posição da opção de escolha do curso";

- com base no teste de independência do quiquadrado, existir uma associação estatisticamente significativa $\quad(\chi 2=7.467, \quad \mathrm{p}=0.024) \quad$ entre $\mathrm{o}$ "Posicionamento face às vivências da praxe" e o "Tempo de estudo". Importa referir que $83.5 \%$ dos estudantes que se posicionaram positivamente face ao papel integrador das praxes referiram estudar 14 horas ou menos por semana, enquanto na mesma situação se encontram apenas $60 \%$ dos estudantes que consideraram o papel das praxes negativo no que se refere à sua integração no Ensino Superior.

- com base no teste de independência do quiquadrado, não existir associação estatisticamente significativa entre o "Posicionamento face às vivências da praxe" e o "Enquadramento familiar de origem".

Em síntese, o "Tempo de estudo" foi a única variável, de entre as estudadas, que apresentou uma associação estatisticamente significativa com o "Posicionamento face às vivências da praxe", em termos do contributo desta para a integração académica. Assim, perspetiva-se que os estudantes que vivenciaram a praxe como um acontecimento que contribuiu para a sua integração na vida académica despenderam mais tempo do que os restantes nestas atividades e investiram menos tempo nos estudos. Contudo, como antes sugerido, este aspeto não parece ter uma repercussão significativa no rendimento académico.

Passa-se a focar a atenção no rendimento académico, uma vez que, como já mencionado, este também pode ser tomado como um indicador de adaptação dos estudantes.

Deste modo, os resultados indicaram que os estudantes cujo curso frequentado foi a sua primeira opção têm um rendimento médio académico superior ao dos restantes (13.30 versus 12.39), sendo as diferenças entre estas médias estatisticamente significativas $(\mathrm{t}=1.988, p=0.049)$. Os estudantes que estudam mais horas por semana (' 15 ou mais horas') têm, conforme expectável, um rendimento académico superior (média $=14.14$ ) ao dos que estudam menos horas (" 6 a 14 horas': média=13.32; e '0 a 5 horas': média=12.13). Importa, ainda, referir que a aplicação da ANOVA a um fator apontou para a rejeição da hipótese da igualdade das médias do rendimento académico nas diferentes categorias da variável "Tempo de estudo" $(\mathrm{F}=9.813$, $p=0.000)$. Os estudantes pertencentes à primeira geração de universitários obtiveram um rendimento académico médio (13.23) ligeiramente mais elevado do que o dos estudantes de geração contínua (12.71), embora as diferenças observadas não sejam estatisticamente significativas $(p>0.05)$. Tal situação pode corresponder à ideia do envolvimento com as atividades académicas como forma de aceder a uma melhor condição de vida (Schuh, 2017).

Foi, ainda, aplicada uma Análise de Variância Fatorial, com o intuito de identificar as variáveis que têm um efeito significativo no "Rendimento académico", entre as quais se incluiu a variável "Posicionamento face às vivências da praxe". Assim, com base na Análise de Variância Fatorial (ANOVA a cinco fatores), considerando como variável dependente o "Rendimento académico" dos estudantes, concluiu-se que as variáveis "Sexo" ( $\mathrm{F}=8.22 ; p=0.006)$, "Tempo de estudo" ( $\mathrm{F}=9.83 ; p=0.000)$ e "Posição da opção de escolha do curso" $(\mathrm{F}=5.93 ; p=0.018)$ têm um efeito significativo no "Rendimento académico" dos estudantes, o mesmo não se podendo afirmar relativamente às variáveis "Enquadramento familiar de 
origem" e "Posicionamento face às vivências da praxe". Os diversos testes de comparações múltiplas aplicados foram unânimes na identificação de diferenças significativas $(p<0.05)$ entre todos os pares de médias das notas (rendimento académico) dos estudantes incluídos nos grupos definidos pelas categorias da variável "Tempo de estudo".

A Tabela 1 é referente à caracterização dos clusters, C1, C2 e C3, resultantes do método das k-médias.

Tabela 1.

Caracterização dos estudantes incluídos em cada um dos clusters

\begin{tabular}{lccc}
\hline \multicolumn{5}{c}{$\mathrm{C} 1$} & $\mathrm{C} 2$ & $\mathrm{C} 3$ \\
\hline \multicolumn{4}{c}{ Género } \\
\hline Masculino & $56.7 \%$ & $46.8 \%$ & $20.0 \%$ \\
Feminino & $43.3 \%$ & $53.2 \%$ & $80.0 \%$ \\
\hline \multicolumn{4}{c}{ Posição da opção de escolha do curso } \\
\hline $1^{\text {a }}$ opção & $66.7 \%$ & $81.6 \%$ & $87.5 \%$ \\
Outra & $33.3 \%$ & $18.4 \%$ & $12.5 \%$ \\
\hline \multicolumn{4}{c}{ Tempo de estudo (horas) } \\
\hline 0 a 5 & $48.3 \%$ & $36.5 \%$ & $15.0 \%$ \\
6 a 14 & $37.9 \%$ & $39.2 \%$ & $47.5 \%$ \\
$15+$ & $13.8 \%$ & $24.3 \%$ & $37.5 \%$ \\
\hline \multicolumn{4}{c}{ Enquadramento familiar de origem } \\
\hline $1^{\text {a geração }} 66.7 \%$ & $79.2 \%$ & $85.0 \%$ \\
Geração contínua & $33.3 \%$ & $20.8 \%$ & $15,0 \%$ \\
\hline Posicionamento face às vivências da praxe \\
\hline Negativo & $13.0 \%$ & $27.8 \%$ & $39.3 \%$ \\
Positivo & $87.0 \%$ & $72.2 \%$ & $60.7 \%$ \\
\hline
\end{tabular}

Os clusters $\mathrm{C} 1, \mathrm{C} 2$ e C3 contêm, respetivamente, $20.4 \%, 52.4 \%$ e $27.2 \%$ do total de inquiridos, sintetizando-se na Tabela 1 as principais características dos estudantes incluídos em cada um destes grupos, de forma a se descrever o perfil dos mesmos.

Os estudantes do cluster C1 (estudantes com rendimento académico mais baixo: média $=10.09$; $\mathrm{DP}=1.177$ ) são predominantemente do sexo masculino e os que estudam menos horas por semana, sendo de salientar que $48.3 \%$ destes afirmaram estudar entre zero a cinco horas. É, ainda, de realçar que é este o cluster que contém uma maior proporção de estudantes que pertencem à geração contínua de universitários (33.3\%) e uma maior proporção de estudantes cujo curso frequentado não é o da sua primeira opção (33.3\%), comparativamente aos outros clusters. Importa, ainda, salientar que é nesta classe que se encontra a maior proporção de estudantes $(87 \%)$ que se posicionam positivamente face ao papel integrador das vivências da praxe.

Nos clusters C2 (estudantes com rendimento académico intermédio: média $=12.88 ; \mathrm{DP}=0.818$ ) e C3 (estudantes com rendimento académico mais elevado: média $=15.84$; $\mathrm{DP}=1.151$ ) predominam os estudantes que, na sua esmagadora maioria, são do sexo feminino, entraram para o curso em que se inscreveram na primeira opção, são estudantes da primeira geração e estudam tendencialmente entre seis a catorze horas. É, ainda, de realçar que o cluster 3, comparativamente aos outros clusters, é o que contém uma maior proporção de estudantes que percecionaram as vivências da praxe de uma forma negativa $(39.3 \%)$ para a sua integração académica.

\section{Discussão}

O "Posicionamento face às vivências da praxe", a nível da integração dos estudantes desta amostra, não reflete estar associado às variáveis dos domínios académico e familiar estudadas neste trabalho. A única variável, entre as estudadas, que diferenciou os estudantes que se posicionaram positivamente vs negativamente face à praxe foi o "Tempo de estudo". Assim, como já mencionado, presume-se que os estudantes que vivenciam a praxe como um acontecimento favorecedor da sua integração na nova academia investem mais tempo nessas atividades e envolvem-se menos nos estudos, pelo menos durante os tempos iniciais de entrada na instituição. Contudo, este menor compromisso com os estudos não parece afetar os resultados finais deste grupo de estudantes, uma vez que o "Rendimento académico" não surge como variável diferencial no que se refere ao "Posicionamento face às vivências da praxe".

Por sua vez, o "Rendimento académico" é influenciado pelas variáveis "Sexo", "Posição de escolha do curso" e "Tempo de estudo". Não se verificaram diferenças significativas entre o rendimento académico dos estudantes pertencentes à primeira geração de universitários na família e os restantes, embora os da primeira geração tenham tido um rendimento académico médio ligeiramente superior. Daqui, conjetura-se que variáveis motivacionais, associadas à escolha e decisão (por exemplo, frequentar o curso que se escolheu como $1^{\mathrm{a}}$ opção) e o envolvimento comportamental, refletido, por exemplo, no número de horas de estudo, são mais influentes a nível do rendimento académico do que o facto de os estudantes terem ou não pelo menos um dos pais com o Ensino Superior.

Estudantes em anos mais avançados poderão apresentar resultados diferentes, pelo que este é um propósito a investigar subsequentemente.

\section{Referências}

Andrade, C. (2016). A construção da Identidade, Autoconceito e Autonomia em Adultos Emergentes. Psicologia Escolar e Educacional, 20(1), 137-146. http://dx.doi.org/10.1590/2175353920150201944

Araújo, A. M., Santos, A. A., Noronha, A. P., Zanon, C., Ferreira, J. A., Casanova, J. R., \& Almeida, L. S. (2016). Dificuldades antecipadas de adaptação ao ensino superior: um estudo com alunos do primeiro ano. Revista de Estudios e Investigación en Psicología y Educación, 3 (2), 102-111. 
Botelho, S., Mendes, M., Caldeira, S. N., Silva, O., Martins, M. J. D., \& Barreto Carvalho, C. (2015). Praxe no ensino superior: Um estudo comparativo entre instituições. Revista de Estudios e Investigación en Psicología y Educación, Extr(2), A2001-A2006. doi: 10.17979/reipe.2015.0.0

Caldeira, S. N., Silva, O., Mendes, M., \& Botelho, S. (2016). Praxes académicas: jovens e desafio de integração no ensino superior. In Rocha, G. P. N. \& R. L. Gonçalves \& P. D. Medeiros (Org.), Juventude(s). Novas realidades, novos olhares (pp. 149-168). V.N. Famalicão: Húmus.

Caldeira, S. N., Silva, O., Mendes, M., Martins, M. J., Miranda, R., \& Silva, N. (2017). A entrada no ensino superior: Uma comparação entre instituições de Portugal e do Brasil. In L. S. Almeida \& R. V. Castro (orgs), Ser estudante no Ensino Superior: As respostas institucionais à diversidade de públicos (pp. 64-81.) Braga: Centro de Investigação em Educação (CIEd) Instituto de Educação, Universidade do Minho. http://www.cied.uminho.pt/uploads/2017-SerEstudante-no-Ensino-Superior.pdf

Costa, S., Dias, O., Dias, A., Souza, T., \& Canela, J. (2013). Trote Universitário: Diversão ou Constrangimento entre os Académicos de Saúde?. Revista Bioética, $21 \quad$ (2), pp. 250-258. http://www.scielo.br/pdf/bioet/v21n2/a19v21n2.pdf

Direção Regional da Educação (2015). ProSucesso Açores pela Educação: Plano Integrado de Promoção do Sucesso Escolar. Acedido em 15 de maio de 2015, de: https://www.azores.gov.pt/NR/rdonlyres/45251 8FA-EE58-40B0-A752-356AF8F63FA3/870503 /ProSucesso.pdf

Martins, M. J., Caldeira, S. N., Silva, O., Mendes, M., \& Botelho, S. P. (2015). O que pensam os estudantes de enfermagem sobre as praxes académicas? In Atas $13^{\circ}$ Colóquio Internacional de Psicologia e Educação, Lisboa: ISPA - Instituto Universitário, 25 a 27 de junho de 2015, pp. 431-446.

Pimentel, M., Mata, M., \& Pereira, F. (2012). Práticas Iniciáticas de Integração no Ensino Superior. Um Ritual Institucionalizado ou um Processo de (des) Integração? Atas do V Encontro do CIED-Escola e Comunidade. Lisboa: Escola Superior de Educação de Lisboa, $\quad$ pp. $\quad 393 \quad$ - $\quad 401$. http://hdl.handle.net/10198/8975

Sarason, I. G., Levine, H. M., Basham R. B., \& Sarason, B. R. (1983). Assessing Social Suport: The Social Support Questionnaire. Journal of Personality and Social Psychology, 44(1), 127-139.

Schuh, M. S. (2017). A trajetória da primeira geração da família na universidade: contribuições acerca da formação acadêmica na PUCRS. Dissertação de Mestrado. Porto Alegra: Pontifícia Universidade Católica do Rio Grande do Sul.

Silva, O., Caldeira, S. N., Mendes, M., \& Botelho, S. (2014). Bullying in Hazing Practices: A Study on Higher Education. In ICERI 2014 Proceedings 7th Conference of Education, Research and Innovation,
Seville: IATED Academy, 17 a 19 de novembro de 2014, pp. 0784-0791

Silva, O., Caldeira, S. N., Mendes, M., Botelho, P. S., \& Martins, J. M. (2015). Caracterização da praxe académica com recurso à análise de dados. In XXII Jornadas de Classificação e Análise de Dados (JOCLAD2015) - Livro de Resumos. Politécnico de Setúbal, Escola Superior de Tecnologia do Barreiro, Barreiro: INE, 10 a 11 de abril de 2015, pp.107-108.

Silva, O., \& Sousa, Á. (2015). (Dis)Similaridades nas Características da População Açoriana. Organizadores: Gilberta Rocha e Álvaro Borralho, Novas e Velhas Tendências Populacionais, Editor: Fernando Mão de Ferro, pp. 21 - 42. Lisboa: Edições Colibri. ISBN: 978-989-689-538-9.

Soares, A., Pinheiro, M., \& Canavarro, J. (2015). Transição e adaptação ao ensino superior e a demanda pelo sucesso nas instituições portuguesas. PSYCHOLOGICA, 58(2), 97-116.

Stephens, N. M., Fryberg, S. A., Markus, H. R., Johnson, C., \& Covarrubias, R. (2012). Unseen disadvantage: How American universities' focus on independence undermines the academic performance of first-generation college students. Journal of Personality and Social Psychology, 102 (6), 11781197.

\section{Agradecimentos}

The first two authors are from the University of the Azores, CICS.UAc/CICS.NOVA.UAc, UID/SOC/04647/2013, and this paper was produced with support from the FCT/MEC thru National Funds and when applied co-financed by the FEDER within the partnership agreement PT2020. 